\title{
Improving Touch Accuracy on Large Tabletops Using Predecessor and Successor
}

\author{
Max Möllers, Norbert Dumont, Stefan Ladwig, Jan Borchers \\ RWTH Aachen University, Germany \\ \{moellers, borchers\}@cs.rwth-aachen.de, stefan.ladwig@psych.rwth-aachen.de, \\ norbert.dumont@rwth-aachen.de
}

\begin{abstract}
Touch interfaces provide great flexibility in designing an UI. However, the actual experience is often frustrating due to bad touch recognition. On small systems, we can analyze yaw, roll, and pitch of the finger to increase touch accuracy for a single touch. On larger systems, we need to take additional factors into account as users have more flexibility for their limb posture and need to aim over larger distances. Thus, we investigated how people perform touch sequences on those large touch surfaces. We show that the relative location of the predecessor of a touch has a significant impact on the orientation and position of the touch ellipsis.
\end{abstract}

We exploited this effect on an off-the-shelf touch display and showed that with only minimal preparation the touch accuracy of standard hardware can be improved by at least $7 \%$, allowing better recognition rates or more UI components on the same screen.

\section{Author Keywords}

touch; tabletops; offset; accuracy; sequence

\section{ACM Classification Keywords}

H.5.2 Information Interfaces and Presentation: Miscellaneous

\section{INTRODUCTION}

Touch interfaces are currently the dominant design in mobile computing, and touch-enabled tabletops might become a major computing platform for knowledge workers in the future. However, bad touch recognition often leaves users with frustration regardless of the surface size as not only small targets but also long distances make touching hard [3]. One can increase the target size to compensate for this effect. Another option that even preserves the precious real estate is to correct the user's input for errors. Existing research already tells us how we can do this for single touches on small surfaces [5], yet the correction of touch (sequences) on large-scale surfaces has not been investigated.

Permission to make digital or hard copies of all or part of this work for personal or classroom use is granted without fee provided that copies are not made or distributed for profit or commercial advantage and that copies bear this notice and the full citation on the first page. To copy otherwise, or republish, to post on servers or to redistribute to lists, requires prior specific permission and/or a fee.

CHI 2013, April 27-May 2, 2013, Paris, France.

Copyright 2013 ACM 978-1-4503-1899-0/13/04...\$15.00.

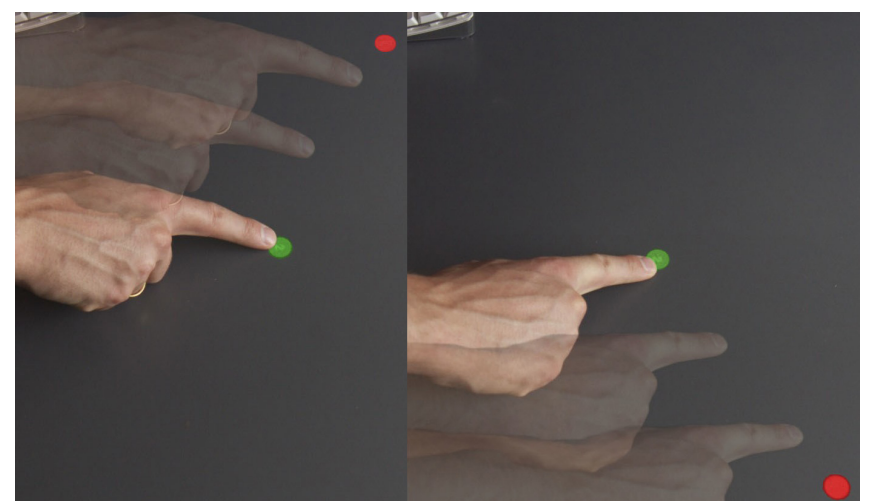

Figure 1. A person hitting the same (green) button, coming from different directions. Although the same target, the person hits it differently.

Also, on larger interaction areas, people have more freedom for touching the surface-different body, arm, finger orientations are possible. However, as people plan actions to be as comfortable as possible [7], we expect a predictable behavior, e.g., changing finger orientation in a sequence of touches depending on the previous or following touch (Fig. 1).

This paper contributes the following: 1) a study on how predecessor and successor location affect touch location and orientation and 2) an application of the predecessor effect to an off-the-shelf touch display showing a significant improvement of accuracy.

\section{RELATED WORK}

Several solutions were proposed to solve the problem of inaccurate input as well as occlusion from the finger itself. Some use indirect manipulation by having an input area act as a proxy for the area which is manipulated. This proxy can be located close to the actual input area (cross-lever, precision handle [1], and shift [8]) or farther away, e.g., back-of-device interactions [2].

Other methods record more than just the touch point to increase accuracy. Wang and Ren [9] also employed the contact ellipsis. TapSense [4] used the sound of the impact of an object to identify the touch. TouchID Toolkit [6] used gloves with fiducial markers on the finger tips, knuckles, etc. to thoroughly identify fingers and their posture. The Ridge Pad is able to track yaw, roll, and pitch from the fingerprint [5].

None of these systems take touch sequences into account. This makes sense for small touch surfaces as only minimal movement inertia is to be expected and limb postures are very 
similar. However, on larger surfaces limb postures can vary a lot and movement inertia comes into play. In action sequences, Rosenbaum et al. [7] analyzed target acquisitions and showed that subjects seem to plan their actions beyond the first grip, anticipating future states.

\section{TOUCH SEQUENCES ON TABLETOPS}

People who work frequently with the same application know which input is required for achieving a goal. They turn this into a sequence of actions, which on a touch device usually consist of a sequence of touches. We assume that individuals plan these sequences of touches by predicting comfortable final limb postures [7].

Thus, given a touch sequence $\left[\ldots, t_{i-1}, t, t_{i+1}, \ldots\right]$ our hypotheses are:

H1 Coming from different touch points $t_{i-1}$ changes the angle and offset of the touch at $t_{i}$. Predecessor Effect (PE).

H2 Going to different touch points $t_{i+1}$ changes the angle and offset of the touch at $t_{i}$. Successor Effect (SE).

We also consider angle (yaw) of the touch ellipsis (Fig. 2, right) as previous work showed a significant effect of this angle on the touch offset.

To evaluate our hypotheses, we let people perform touch sequences on a tabletop in a controlled setting and analyzed our data regarding the $\mathrm{PE}$ and the SE.

\section{Setup}

We used a 6-ring plus middle button layout for the touch targets (Fig. 2, left). The northernmost button was reachable with no arm stretching or leaning forward. We used round buttons (15 mm diameter) and the ring had a diameter of 40 $\mathrm{cm}$. Dependent on the study condition participants performed up to three touches in a sequence. Only buttons used in the sequence were displayed. These buttons were labeled with "1" to "3" (Fig. 2, right), colored gray, and lit up in green color when they needed to be touched. Users were asked to move their finger to a pre-defined position after performing one touch sequence: a $10 \mathrm{~cm}$ by $5 \mathrm{~cm}$ wide box on the left half of the table, easily reachable with the right hand.

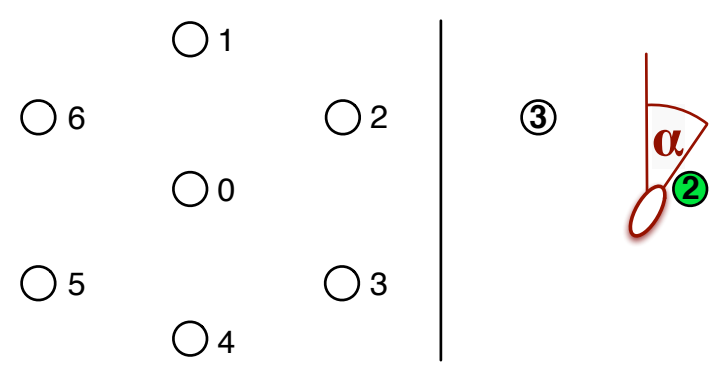

Figure 2. Left) Layout of the buttons in our study. Most touch sequences started on the ring, went to its center and back to the ring. We only look at touches aimed at the center. Right) A participant needs to touch the buttons labeled with " 1 " to " 3 ": After touching the outer button, he aims at the center button, but overshoots (red ellipsis). We record the location and orientation $\alpha$ w.r.t. the $\mathbf{y}$-axis.

Our experiment used four conditions (cf. video figure):
A first, middle, and last button are displayed simultaneously, i.e., PE and SE should occur.

B first and second are displayed directly at the beginning, then third after the second has been touched, i.e., PE should occur, but SE not as the participant does not know the successor when touching.

$\mathrm{C}$ first is not displayed at all, middle and last are displayed directly at the beginning, i.e., PE does not occur as there are no different predecessors (the resting spot is always the same), but SE should happen.

D first is not displayed at all, middle is displayed directly at the beginning, the last after the middle has been touched, i.e., PE and SE should not happen.

Conditions $\mathrm{A}$ and $\mathrm{B}$ presented sequences of three touches each. Conditions $\mathrm{C}$ and $\mathrm{D}$ presented sequences of two touches each. Users started with doing one condition, performed latin square balanced order of all touch sequences in this condition, repeated 5 times, and then went to the next condition. This order of the conditions was also latin square balanced among the users.

\section{Task}

First, the experimenter gave instructions on how to perform the task and participants were told that their speed and accuracy was a non-important factor. All users were sat at the same predefined position in front of the table and were instructed to use their index finger for touching.

We started the test with a whack-a-mole game for about five minutes in advance of each experiment. This way, participants were able to familiarize themselves with the setting. Then the first touch sequence was presented and participants were asked to press the lit buttons according to their occurrence. Participants took 30 minutes including preparation.

\section{Apparatus \& Analysis}

We use a multi-touch table with a $3240 \times 1920$ px display over $140 \times 80 \mathrm{~cm}$. For accurate touch tracking, we use a single camera beneath the table that can be rotated along its axes to be pointed at a specific spot on the screen. This gives us an input resolution of 140 dpi, i.e., sub-pixel accurate tracking for our 59 dpi output resolution.

We collected demographic data before the test using a questionnaire: gender, age, height, and prior experience with touch devices (5-point Likert scale). Our system returned the touch as a contact ellipsis. We record the angle of its main axis and the center of the ellipsis.

We had concerns how the participants height would impact the body posture which might lead to different touch angles. However, arm length scales with height and seemed to counterbalance this effect: we could not see any effect of height.

\section{Participants}

14 right-handed people ( 2 females) took part in the study, aged $20-31$, height $163-193(M=181, S D=7.6)$. The questionnaire revealed that participants had experience using 
small touch devices such as smartphones $(M=3.8, S D=$ $1.2)$ but only few had experience with larger touch displays like tables $(M=1.9, S D=1.4)$.

\section{Results}

To analyze for the PE, we ran a repeated measures one-way ANOVA using data from condition B (where only PE should happen) (Fig. 3, 4, 5). We could see a significant main effect of predecessor on angle $\left(F_{5,60}=22.367, p<0.001\right)$, $\mathrm{x}$-offset $\left(F_{5,60}=12.082, p<0.001\right)$, and y-offset $\left(F_{5,60}=\right.$ $6.830, p<0.001)$. Similar results could be seen in condition A (where predecessor and successor should happen): a significant main effect of predecessor on angle $\left(F_{5,60}=\right.$ $26.680, p<0.001)$, x-offset $\left(F_{5,60}=13.793, p<0.001\right)$, and y-offset $\left(F_{5,60}=7.372, p<0.001\right)$.

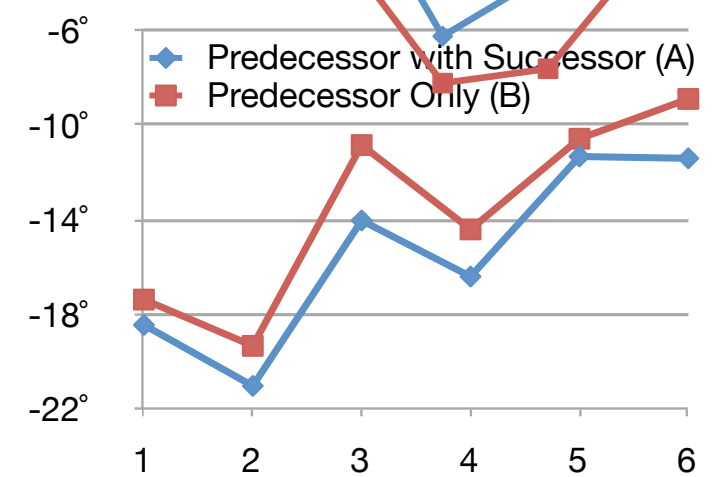

Figure 3. Finger orientation (yaw) depending on the predecessor. It variates similarly in both conditions.

To analyze for the SE, we ran a repeated measures one-way ANOVA using data from condition C (where only SE should happen). It showed no significant main effect of the successor on angle $\left(F_{5,60}=1.567, p=0.183\right)$, x-offset $\left(F_{5,60}=\right.$ $1.150, p=0.344)$, or y-offset $\left(F_{5,60}=0.932, p=0.467\right)$. Similar results were seen in the mixed condition A, i.e., no significant main effects.

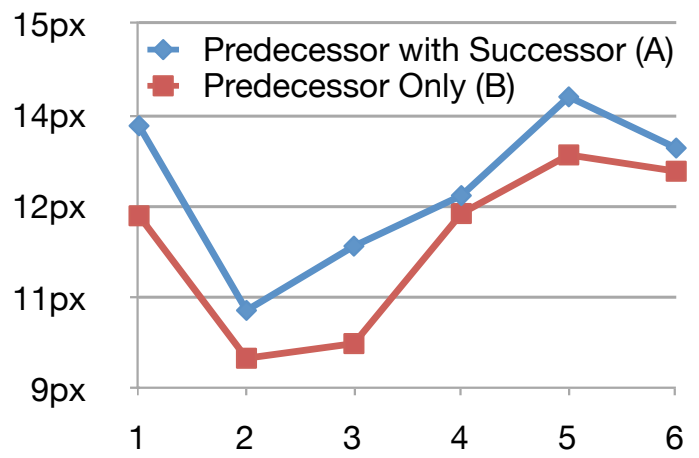

Figure 4. $\mathrm{X}$ offset depending on the predecessor. It variates similarly in both conditions.

As expected in the control condition D, neither predecessor nor successor showed any significant main effect.

\section{Discussion}

Based on the results, we see that there exists a predecessor effect, i.e., the location of a previous touch in a sequence has an impact on future touches. But we didn't see an effect of succeeding touches. This might be due to the fact that this effect is very small or does not exist. In both cases, we actually welcome this result: We do not need to predict user behavior to get very accurate touches.

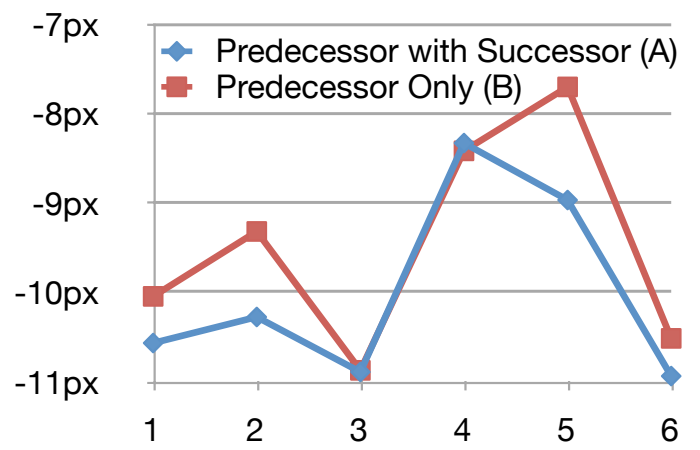

Figure 5. Y offset depending on the predecessor. It variates similarly in both conditions.

Compared to Holz's work, we saw a very low correlation between angle and touch offset $(|r|<0.1$, cf. Fig. 3 to 4, 5 ). Thus, we cannot use the yaw angle of the finger to correct offset, but instead need to use the predecessor in this setting.

\section{APPLICATION OF THE PREDECESSOR EFFECT}

We showed that the predecessor effect exists. We now apply it to improve the touch accuracy on an off-the-shelf touch display using a simple machine learning approach.

\section{Study Setup}

We used a 27" Perceptive Pixel horizontal display with sub pixel accurate tracking on a 110dpi screen. As warm-up, we sat the participants in a fixed spot in front of the display, displayed a round button, and asked them to touch the button 30 times with fixed pitch ("close to horizontal") and roll ("straight on the surface"). Between each touch, they should move their finger to a resting position above the table.

Again, we used a 6-ring plus middle button layout for our application. Users touched a button on the ring first and then touched the middle button. After this, they needed to move their hand to a resting position in front of them in the air. The order of the six buttons was counterbalanced and the full set was repeated 15 times. We measured touch location $\vec{t}$ and angle $\alpha$, also per participant.

Similar to other machine learning approaches, the data was split randomly and used in two ways: Two thirds were used to generate a model, the other third was used for evaluation.

\section{Offset Model}

We correct touches according to which direction they come from as we know that the predecessor has an effect on the touch offset: We take all the touches $\vec{t}$ and put them into 6 buckets according to their predecessor $j$ (cf. Algorithm 1). 
For each bucket $B U C K_{j}$, we then take the median $x$ and median $y$ value of the offsets of the touches, resulting in touch correction vectors $\vec{t}_{B U C K_{j}}$. They indicate how people typically over- or under-shoot. We chose to use median instead of the average as the former is more outlier-resistant.

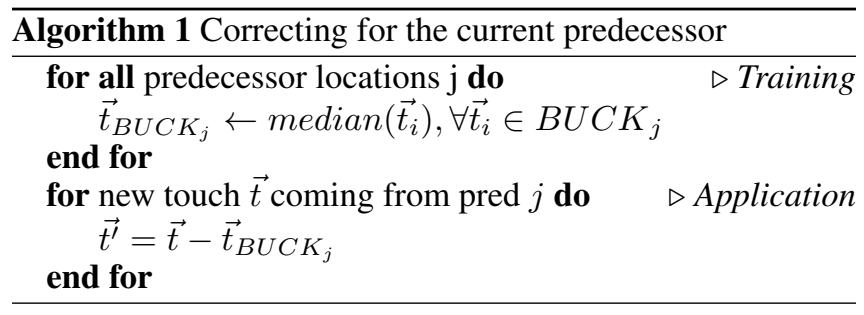

To evaluate, we subtract the correction term according to the model and get $\overrightarrow{t^{\prime}}$. The correction term can also be based only on per participant data to account for individual behavior. This results in two simple look-up tables.

\section{Participants \& Analysis}

We recruited 10 right-handed participants ( 1 female), aged 24-32 $(M=27.1, S D=2.9)$. The questionnaire revealed that participants had experience using small touch devices such as smartphones $(M=4.5, S D=0.7)$ and little experience using table-sized touch systems $(M=2.8, S D=1.4)$. Each participant performed the study in less than 10 minutes.

We create two datasets based on the touch data: corrected for the predecessor as well as the corrected for predecessor and user. For comparison, we calculate the size of a minimal rectangular button that covers at least $95 \%$ of the touches, i.e., its width and height is equal to about 1.96 standard deviations of the mean of the $x$ and $y$ data.

\section{Results}

Only using the predecessor has no effect, neither good nor bad, on the button size (Fig. 6). Our explanation is that individual differences in touch behavior overshadow the predecessor effect. However, taking individual behavior into account, we can shrink the button by $7 \%$ - and we only needed to capture five minutes of user interaction.

\section{FUTURE WORK}

We would like to vary more of our parameters: button sizes and shapes, relative user location, targeting speed, etc. We also want to investigate which time is the right threshold to decide whether a touch can affect the next one. Also, more predecessors could explain the sine shape of Fig. 4 and 5.

In a real application, we would extend our 6-ring to a model that stores offset per relative direction (in degrees) and relative distance to every predecessor. This model could even be stored per each pixel to account for potential differences due to the relative location to the user. As this would be a rather big model and thus hard to calibrate beforehand, we want to explore ways to learn this model during the systems usage, similar to what current smartphones do to correct for touch errors and personal touch behavior.

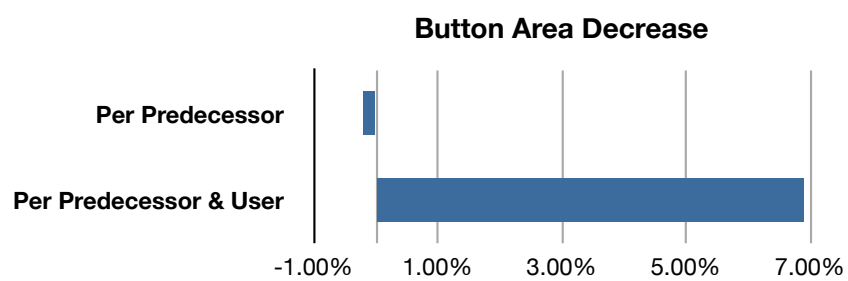

Figure 6. Decrease of minimum button size to allow for $95 \%$ touch accuracy. The baseline was the size of a button using raw touch data.

\section{CONCLUSION}

We evaluated how touch sequences have an effect on the accuracy of their single touches. We saw that the location of the previous touch affects the location and orientation of the following touch. We applied this knowledge to an off-theshelf touch table and were able to improve its accuracy with less than 5 minutes of per participant setup. It is quite likely that even this short learning phase can be performed during systems usage, making no setup necessary while improving touch accuracy for any large touch surface.

\section{ACKNOWLEDGMENTS}

This work was funded in part by the German B-IT Foundation.

\section{REFERENCES}

1. P. Albinsson and S. Zhai. High precision touch screen interaction. Proc. CHI '03, 105-112.

2. P. Baudisch. Back-of-device interaction allows creating very small touch devices. Proc. CHI '09, 1923-1932.

3. P. M. Fitts. The information capacity of the human motor system in controlling the amplitude of movement. Journal of Experimental Psychology, 47(6):381-391, 1954.

4. C. Harrison, J. Schwarz, and S. E. Hudson. TapSense: enhancing finger interaction on touch surfaces. Proc. UIST' '11, 627-634, October.

5. C. Holz and P. Baudisch. The generalized perceived input point model and how to double touch accuracy by extracting fingerprints. Proc. CHI' 10, 581-590.

6. N. Marquardt, J. Kiemer, D. Ledo, S. Boring, and S. Greenberg. Designing user-, hand-, and handpart-aware tabletop interactions with the TouchID toolkit. Proc. ITS '11, 21-30, November.

7. D. A. Rosenbaum, F. Marchak, H. J. Barnes, J. Vaughan, J. D. Slotta, and M. J. Jorgensen. Constraints for action selection: Overhand versus underhand grips. Lawrence Erlbaum Associates, Inc, Mahwah, New Jersey, 1990.

8. D. Vogel and P. Baudisch. Shift: a technique for operating pen-based interfaces using touch. Proc. CHI '07, 657-666, April.

9. F. Wang and X. Ren. Empirical Evaluation for Finger Input Properties In Multi-touch Interaction. Proc. CHI '09, 1063-1072. 